\title{
Propuesta metodológica para la solución de ecuaciones polinómicas en el campo de los números complejos
}

(c)(1)(8)

Methodological proposal for the solution of polynomial equations in the field of complex numbers

Rómel Manolo Insuasti Castelo. ${ }^{1} \&$ Javier Roberto Mendoza Castillo. ${ }^{2}$

\begin{abstract}
.
DOI: https://doi.org/10.33262/concienciadigital.v4i3.1.1830

Introduction. On many occasions, in solving different technical problems, the task of solving polynomial equations of degree $\mathrm{n}$ is presented, in which it is necessary to find the roots of these. Within the probabilities you can have real or complex roots, depending on the characteristics of the polynomial in question. The real ones that are widely used because there is a notion of said values and applicable in real problems. On the other hand, complex roots that, as they exist, do not have a real notion, which sometimes are not considered in the solution of real problems, but on many occasions if they are necessary to consider them, as is the case of the solution of differential equations, among other applications, this implies that these roots must be found. Objective. Propose a methodology that facilitates the calculation of complex roots in polynomial equations. Methodology. The research design is of the quantitative cross-sectional type. It is based fundamentally on a methodological calculation proposal of the roots of polynomial equations of degree $n$, which implies a combination of different numerical methods and the respective conceptualization to find both real and complex roots, as intermediate

1 Escuela Superior Politécnica de Chimborazo, Facultad de Mecánica. Riobamba, Ecuador. rinsuasti@espoch.edu.ec, https://orcid.org/0000-0002-4170-1511

2 Escuela Superior Politécnica de Chimborazo, Facultad de Recursos Naturales. Riobamba, Ecuador. jmendoza@espoch.edu.ec, https://orcid.org/0000-0003-3148-0193
\end{abstract}


information for the solution of later problems. Results. Once the proposed methodology is applied, its feasibility in terms of solving a polynomial equation is analytically demonstrated. Conclusion. The procedure used is based on the operations of complex numbers, since it is necessary to evaluate the functions defined for the polynomial as well as for its derivative with complex numbers.

Keywords: Methodology, solution of polynomial equations, numerical methods.

\section{Resumen.}

Introducción. En muchas ocasiones en la solución de diferentes problemas técnicos se presenta la tarea de resolver ecuaciones polinómicas de grado n, en las cuales se necesita encontrar las raíces de estas. Dentro de las probabilidades se pueden tener raíces reales o complejas, dependiendo de las características del polinomio en cuestión. Las reales que son muy utilizadas pues se tiene una noción de dichos valores y aplicables en los problemas reales. Por otra parte, las raíces complejas que, al existir, estas no tienen una noción real, las cuales en ocasiones no son consideradas en la solución de los problemas reales, pero en muchas ocasiones si son necesarias considerarlas, como es el caso de la solución de ecuaciones diferenciales, entre otras aplicaciones, esto implica que se deben encontrar dichas raíces. Objetivo. Proponer una metodología que facilite el cálculo de las raíces complejas en ecuaciones polinómicas. Metodología. El diseño de la investigación es del tipo cuantitativa de corte transversal. Se basa fundamentalmente en una propuesta metodológica de cálculo, de las raíces de ecuaciones polinómicas de grado n, la cual implica una combinación de diferentes métodos numéricos y la conceptualización respectiva para ir encontrando las raíces tanto reales como complejas, como información intermedia para la solución de problemas posteriores. Resultados. Una vez aplicada la metodología propuesta se demuestra analíticamente su factibilidad en cuanto a la resolución de una ecuación polinómica. Conclusión. El procedimiento empleado se basa en las operaciones de números complejos, pues es necesario evaluar las funciones definidas para el polinomio como para su derivada con números complejos.

Palabras claves: Metodologia, solución ecuaciones polinómicas, métodos numéricos.

\section{Introducción.}

La solución de ecuaciones polinómicas como un proceso intermedio en la solución de problemas técnicos reales, reviste el encontrar las raíces, estas dependiendo del polinomio pueden ser reales o complejas, existen algunas aplicaciones en las cuales son necesarias únicamente la raíces reales, pero en otras ocasiones como al resolver ecuaciones diferenciales es necesario encontrar todas la raíces reales y complejas pues estas son partes de la solución de la ecuación diferencial, en términos generales cuando es necesario encontrar todas la raíces del polinomio, en ocasiones se dificulta por que los procesos o la metodología de solución implica la experiencia del que resuelve la ecuación, esto debido a que se tiene que resolver por métodos convencionales como el de factorización, 
Ruffini entre otros, los cuales en ocasiones no son tan fáciles para encontrar las soluciones. Por esta razón en este estudio se pretende generalizar la resolución de una ecuación polinómica de grado $\mathrm{n}$, con la ayuda de métodos numéricos y de conceptualizaciones relacionadas para de esta manera simplificar el grado de la ecuación e ir reduciéndola hasta encontrar todas las raíces sean reales o complejas.

\section{Metodologia.}

La metodología a ser utilizada en este estudio está basada en métodos numéricos con un tratamiento general de números complejos, lo cual permite encontrar raíces de valores reales como complejos.

Una ecuación polinómica de grado n, tiene la forma general.

$$
a_{0} x^{n}+a_{1} x^{n-1}+a_{2} x^{n-2}+\cdots+a_{n-1} x+a_{n}=0
$$

Donde: $a_{0}, a_{1}, a_{2}, \ldots, a_{n}$ son coeficientes constantes y $a_{0} \neq 0$

Otra forma de determinar una ecuación polinómica es:

$$
P(x)=a_{0} x^{n}+a_{1} x^{n-1}+a_{2} x^{n-2}+\cdots+a_{n-1} x+a_{n}=0
$$

Donde $P(x)$ es el polinomio de grado n en la variable $x$ y en una ecuación polinómica su valor es cero.

Se conoce que las raíces de una ecuación son los valores que al evaluar en la ecuación cumplen con la igualdad.

Se entiende que el número de raíces de dicha ecuación es igual al grado de la ecuación polinómica, teniendo en cuenta que estas raíces pueden ser reales y complejas. Autores como: Ochoviet C., Martínez G., Galán N., Bagni, G. Randolph, V, Macías D., concuerdan que en determinados problemas solo es necesario determinar las raíces reales e incluso se pueden considerar únicamente las raíces positivas, pero lo que nos ocupa es determinar todas las raíces pues algunos procesos así lo requieren.

Por lo tanto, en forma general las raíces tendrán la forma de un número complejo:

$$
x=a \pm b i
$$

Donde $a$ es la parte real de la raíz y bi es la parte imaginaria de la misma, aquí se puede observar que cuando $b=0$, entonces se tiene una raíz real, mientras si $a=0$, entonces tenemos una raíz imaginaria.

Se debe aclarar que las raíces presentes en este tipo de ecuaciones dependiendo el grado del polinomio se puede tener varias posibilidades en relación a que las raíces pueden ser reales y/o complejas, como se detalla en la siguiente tabla: 
Tabla 1.

Referencias de raíces de acuerdo con el grado de polinomio

\begin{tabular}{ccc}
\hline Grado de la ecuación & Raíces reales & Raíces imaginarias \\
\hline 1 & 1 & \\
\hline 2 & 2 & 2 \\
\cline { 2 - 3 } & 0 & 0 \\
\hline 4 & 3 & 2 \\
\hline 4 & 1 & 0 \\
\hline 5 & 4 & 2 \\
\hline & 2 & 4 \\
\hline & 0 & 0 \\
\hline & 5 & 2 \\
\hline
\end{tabular}

Fuente: Elaboración propia

En términos generales podemos decir que las raíces complejas siempre se las calcula por pares y son de la forma $x=a \pm b i$.

La solución de la ecuación polinómica se la puede resolver por factorización, lo cual resulta difícil en un polinomio de grado n, la otra opción es utilizar Ruffini para determinar la raíz, pero también resulta difícil ubicar la raíz, pues se tiene un sin número de valores a evaluar. Más aun cuando las raíces son imaginarias. Por lo tanto, se hace necesario un procedimiento para resolver este tipo de ecuaciones definiendo en forma ordenada como proceder para obtener resultado óptimo y en corto tiempo. (Benjumea, Fernández, \& Márquez, 2006)

\section{Análisis y Propuesta}

El objetivo de resolver una ecuación polinómica es la de encontrar las raíces, dependiendo de grado de la ecuación tenemos claro cuantas soluciones debemos encontrar, algo de lo que se puede estar seguro es que si el grado del polinomio es impar existe por lo menos un raíz real, lo cual guiara el procedimiento a seguir, si el grado es par habrá que probar si las raíces son reales o complejas, si en el proceso se encuentra una raíz real, esto implica que debe existir por lo menos una raíz más real. (Muto, 2021)

Como hemos indicado la ecuación polinómica tiene la forma siguiente

$$
a_{0} x^{n}+a_{1} x^{n-1}+a_{2} x^{n-2}+\cdots+a_{n-1} x+a_{n}=0
$$

Donde: $a_{0}, a_{1}, a_{2}, \ldots, a_{n}$ son coeficientes constantes y $a_{0} \neq 0$

Se propone resolver por el método numérico de Newton Rampson el cual nos da una rápida convergencia tanto para raíces reales como complejas. Por lo que es necesario introducir la metodología para utilizar dicho método. Se debe aclarar que este método 
sirve para resolver cualquier tipo de ecuación principalmente las no lineales, por esta razón podemos aplicar en las ecuaciones polinómicas. (Rodríguez, 2017)

En general si consideramos al polinomio como una $f(x)$, se puede asociar a una curva en el plano. En donde con un valor inicial $x_{i}$ se encuentra el correspondiente valor sobre la función que sería $f\left(x_{i}\right)$, que genera un punto $\left(x_{i} f\left(x_{i}\right)\right)$, sobre este punto en la curva trazamos una recta tangente cuya intersección con el eje $\mathrm{x}$ genera un punto $\left(x_{i+1}, 0\right)$, estos dos puntos permite definir a la ecuación de la recta, la cual nos interesa el valor de $x_{i+1}$ que es el valor que se aproxima al valor de la raíz, este nuevo valor se lo puede calcular con la ecuación siguiente:

$$
x_{i+1}=x_{i}-\frac{f\left(x_{i}\right)}{f^{\prime}\left(x_{i}\right)}
$$

En donde $f^{\prime}\left(x_{i}\right)$, corresponde a la derivada de $f\left(x_{i}\right)$.

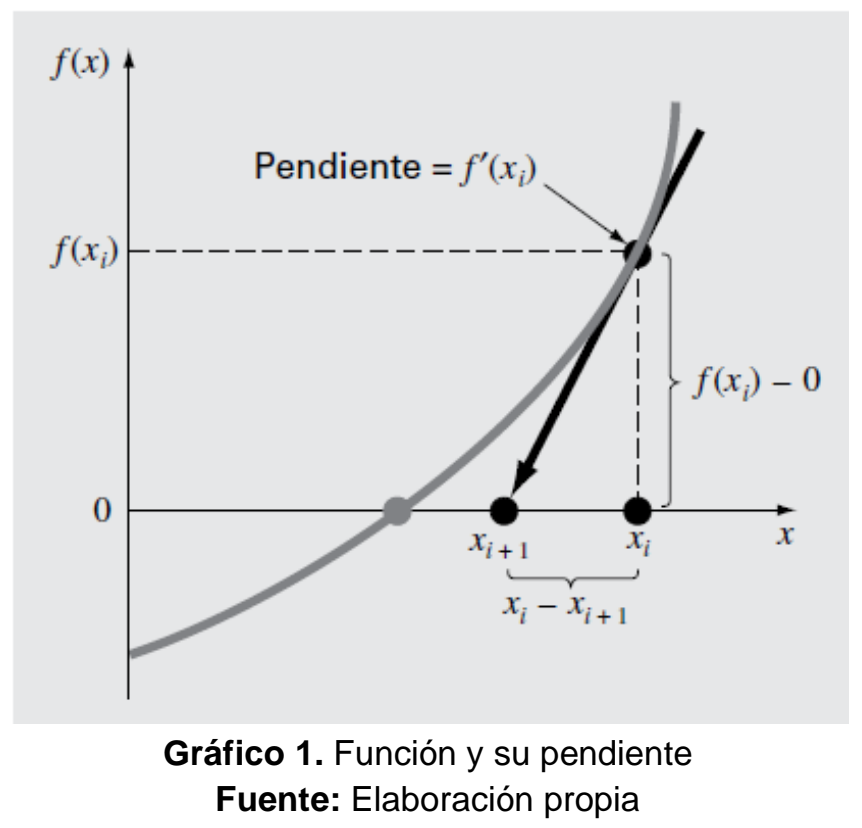

Al ser un método numérico el valor calculado $x_{i+1}$ pasará a ser el valor inicial, cuantas veces sea necesaria hasta que el valor de $x_{i+1} \approx x_{i}$ que en cálculo se puede definir con un valor de convergencia equivalente a $\left|x_{i+1}-x_{i}\right| \leq \varepsilon$, donde $\varepsilon$ es la precisión del cálculo de la raíz. (Ndjatchi 2019).

En el momento que encontramos una raíz real, debemos reducir el grado de la ecuación polinómica en un grado dividiendo la ecuación polinómica por el factor que corresponde a la raíz, de la siguiente manera:

Raíz real: $x=r_{1}$

factor correspondiente: $x-r_{1}=0$

La división a realizar será:

$$
\frac{a_{0} x^{n}+a_{1} x^{n-1}+a_{2} x^{n-2}+\cdots+a_{n-1} x+a_{n}}{x-r_{1}}
$$


El resultado de esta división nos dará como resultado un polinomio de grado n-1, el cual nuevamente entrará a ser evaluado para encontrar una nueva raíz iniciando el método numérico para el nuevo polinomio.

El proceso se repetirá hasta encontrar todas las raíces reales, pero si estas dejan de existir entonces habrá que evaluar para valores complejos es decir de la forma $x=a \pm b i$, para lo cual se suguiere el valor inicial de $x_{o}=1+i$, asegurando que el resultado de $x_{i+1}=$ $x_{i}-\frac{f\left(x_{i}\right)}{f^{\prime}\left(x_{i}\right)}$

Sea también un número complejo. Al encontrar una raíz compleja se entiende que tiene una raíz que es conjugada de esta, por lo que se puede calcular el polinomio restante si se divide el polinomio para el valor que corresponde al resultado del producto de las dos raíces complejas:

$$
\begin{gathered}
(x-a-b i)(x+a+b i)=\left(x^{2}+2 a x+a^{2}+b^{2}\right) \\
\frac{a_{0} x^{n}+a_{1} x^{n-1}+a_{2} x^{n-2}+\cdots+a_{n-1} x+a_{n}}{x^{2}+2 a x+a^{2}+b^{2}}
\end{gathered}
$$

Reduciéndose el polinomio en dos grados, para continuar con la siguiente aproximación a la raíz.

\section{Resultados.}

Se resuelve un polinomio de grado 5 de forma ilustrativa para reforzar la aplicación del método de cálculo.

$$
f(x)=-2 x^{5}+3 x^{4}-x^{3}-3 x^{2}+4 x-7=0
$$

Su derivada es:

$$
f^{\prime}(x)=-10 x^{4}+12 x^{3}-3 x^{2}-6 x+4
$$

Al ser un polinomio de grado impar, existe una raíz real, por lo tanto, encontramos esta raíz dando el valor de $x_{0}=-2$. La tabla resume el cálculo de aproximación.

Tabla 2.

Valores de primera aproximación

\begin{tabular}{cccc}
\hline $\boldsymbol{x}_{\mathbf{0}}$ & $\boldsymbol{f}\left(\boldsymbol{x}_{\mathbf{0}}\right)$ & $\boldsymbol{f}^{\prime}\left(\boldsymbol{x}_{\mathbf{0}}\right)$ & $\boldsymbol{x}_{\mathbf{1}}$ \\
\hline-2 & 93 & -252 & -1.63095238 \\
\hline-1.63095238 & 27.14143823 & -117.01071525 & -1.39899552 \\
\hline-1.39899552 & 6.48025769 & -64.64063384 & -1.29874499 \\
\hline-1.29874499 & 0.86079330 & -48.00633413 & -1.28081416 \\
\hline-1.28081416 & 0.02384778 & -45.36235348 & -1.28028845 \\
\hline
\end{tabular}

Fuente: Elaboración propia

Se acepta como raíz el ultimo valor de $x_{1}=-1.28028845$ y el factor será 
$(x+1.28028845)$. Por lo tanto al dividir el polinomio inicial para el factor que contiene la raíz, se obtiene el polinomio que se debe seguir calculando las nuevas raíces.

$$
\begin{aligned}
& \frac{-2 x^{5}+3 x^{4}-x^{3}-3 x^{2}+4 x-7}{x+1.28028845} \\
& =-2 x^{4}+5.5606 x^{3}-8.1191 x^{2}+7.3948 x-5.4675
\end{aligned}
$$

Este Polinomio entra nuevamente a ser calculado pero esta vez lo hacemos con un valor inicial complejo el cual se recomienda sea $x_{o}=1+i$, obteniendo las siguientes aproximaciones, los cálculos se deben hacer en el campo de los números complejos.

$$
f(x)=-2 x^{4}+5.5606 x^{3}-8.1191 x^{2}+7.3948 x-5.4675=0
$$

Su derivada es:

$$
f^{\prime}(x)=-8 x^{3}+16.682 x^{3}-16.238 x+7.3948
$$

Tabla 3.

Valores de aproximación $1+\mathrm{i}$

\begin{tabular}{clll}
\hline $\boldsymbol{x}_{\mathbf{0}}$ & \multicolumn{1}{c}{$\boldsymbol{f}\left(\boldsymbol{x}_{\mathbf{0}}\right)$} & \multicolumn{1}{c}{$\boldsymbol{f}^{\prime}\left(\boldsymbol{x}_{\mathbf{0}}\right)$} & \multicolumn{1}{c}{$\boldsymbol{x}_{\mathbf{1}}$} \\
\hline $1+\mathrm{i}$ & $-1.1939+2.2778 \mathrm{i}$ & $7.1566+1.1254 \mathrm{i}$ & $1.113956+0.663800 \mathrm{i}$ \\
\hline $1.113956+0.663800 \mathrm{i}$ & $-1.135799+0.281915 \mathrm{i}$ & $3.377852-$ & $1.316009+0.791941 \mathrm{i}$ \\
& & $3.537479 \mathrm{i}$ & \\
\hline $1.316009+0.791941 \mathrm{i}$ & $0.448700-0.159849 \mathrm{i}$ & $6.029061-$ & $1.271383+0.766406 \mathrm{i}$ \\
& & $7.031758 \mathrm{i}$ & \\
\hline $1.271383+0.766406 \mathrm{i}$ & $0.026825-0.013818 \mathrm{i}$ & $5.398141-$ & $1.267915+0.765070 \mathrm{i}$ \\
& & $6.066257 \mathrm{i}$ & \\
\hline $1.267915+0.765070 \mathrm{i}$ & $0.000106-0.000101 \mathrm{i}$ & $5.36469287-$ & $1.267897+0.765069 \mathrm{i}$ \\
& & $5.995046 \mathrm{i}$ & \\
\hline
\end{tabular}

Fuente: Elaboración propia

La raíz hallada es $(x-1.267897-0.765069 i)$, al ser una raíz compleja se deduce que la otra raíz será $(x-1.267897+0.765069 i)$, por lo tanto, dividimos el Polinomio calculado para el resultado del producto de los factores anteriores

$$
\frac{-2 x^{4}+5.5606 x^{3}-8.1191 x^{2}+7.3948 x-5.4675}{x^{2}-2.5358 x+2.1929}=-2 x^{2}+0.489 x-2.4933
$$

Finalmente, para este caso tendríamos que resolver la función

$$
f(x)=-2 x^{2}+0.489 x-2.4933=0
$$

Su derivada es:

$$
f^{\prime}(x)=-4 x+0.489
$$


Tabla 4.

Valores de aproximación final

\begin{tabular}{cccc}
\hline $\boldsymbol{x}_{\mathbf{0}}$ & $\boldsymbol{f}\left(\boldsymbol{x}_{\mathbf{0}}\right)$ & $\boldsymbol{f}^{\prime}\left(\boldsymbol{x}_{\mathbf{0}}\right)$ & $\boldsymbol{x}_{\mathbf{1}}$ \\
\hline $1+\mathrm{i}$ & $-2.0043-3.511 \mathrm{i}$ & $-3.511-4 \mathrm{i}$ & $0.255798+0.847852 \mathrm{i}$ \\
\hline $0.255798+0.847852 \mathrm{i}$ & $-1.061375-0.452916 \mathrm{i}$ & $-0.534192-$ & $0.077381+1.132709 \mathrm{i}$ \\
& & $3.391407 \mathrm{i}$ & \\
\hline $0.077381+1.132709 \mathrm{i}$ & $0.098622+0.203293 \mathrm{i}$ & $0.179475-$ & $0.121319+1.109201 \mathrm{i}$ \\
& & $4.530835 \mathrm{i}$ & \\
\hline $0.121319+1.109201 \mathrm{i}$ & $-0.002756+0.004131 \mathrm{i}$ & $0.003725-$ & $0.122251+1.109822 \mathrm{i}$ \\
& & $4.436806 \mathrm{i}$ & \\
\hline $0.122251+1.109822 \mathrm{i}$ & $-9.664 \mathrm{E}-07-2.312 \mathrm{E}-$ & $-2.083 \mathrm{E}-06-$ & $0.122250+1.109822 \mathrm{i}$ \\
& $06 \mathrm{i}$ & $4.439287 \mathrm{i}$ & \\
\hline
\end{tabular}

Fuente: Elaboración propia

La raíz hallada es $(x-0.122250-1.109822 \mathrm{i})$, al ser una raíz compleja se deduce que la otra raíz será $(x-0.122250+1.109822 \mathrm{i})$, de esta manera completamos todas las raíces del polinomio, como sigue:

$$
\begin{gathered}
x_{1}=-1.28028845 \\
x_{2}=1.267897+0.765069 i \\
x_{3}=1.267897-0.765069 i \\
x_{4}=0.122250+1.109822 \mathrm{i} \\
x_{5}=0.122250-1.109822 \mathrm{i}
\end{gathered}
$$

\section{Conclusiones.}

- La aplicación de la metodología propuesta permite calcular las raíces de polinomios de grado $\mathrm{n}$ en forma precisa, ya sean raíces reales o raíces complejas, si las raíces son complejas estas vienen en pares, ya que estas son conjugadas, razón por la cual se las debe considerar en el procedimiento de solución.

- Para calcular raíces reales el valor inicial debe ser un número real, el cual dependiendo de la cercanía de la raíz convergerá más rápidamente y para raíces complejas el valor inicial debe ser un número complejo el cual se recomienda que se $1+\mathrm{i}$.

- El procedimiento empleado se basa en las operaciones de números complejos, pues es necesario evaluar las funciones definidas para el polinomio como para su derivada con números complejos.

- Se debe tener presente el número de raíces a obtener según el polinomio y las posibles raíces reales y complejas, para ir definiendo el proceso de evaluación. 


\section{Referencias bibliográficas.}

Bagni, Giorgio Tomaso (2001). La introducción de la historia de las matemáticas en la enseñanza de los números complejos. Una investigación experimental desempeñada en la educación media. Revista Latinoamericana de Investigación en Matemática Educativa, RELIME, 4(1) pp. 45-61. Disponible en: https://www.redalyc.org/articulo.oa?id=33540103

Benjumea, J., Fernández, D., \& Márquez, M. (2006). Matemáticas avanzadas y estadística para ciencias e ingenierías. Sevilla: Secretariado de publicaciones de la Universidad de Sevilla.

Galván Navarro, Andrés A. (2005). Solución de Ecuaciones Polinomiales. Investigación y Ciencia, 13(33),50-55. Disponible en: https://www.redalyc.org/articulo.oa?id=67403308

Macías Díaz, Jorge Eduardo (2015). Introducción a las ecuaciones. Investigación y Ciencia, 23(66),76-77. Disponible en: https://www.redalyc.org/articulo.oa?id=67446014012

Martínez Sierra, Gustavo; Antonio Antonio, Rocío (2009). Una construcción del significado del número complejo Revista Electrónica de Investigación en Educación en Ciencias, 4 (1) pp. 1-9. Disponible en https://www.redalyc.org/pdf/2733/273320453002.pdf

Muto, V. (1998). Introducción a los métodos numéricos. Editorial Euskal Herriko Unibertsitatea, Argitalpen Zerbitzua. Bilbao

Ndjatchi, Mbe Koua Christophe (2019). Conocimientos previos de números complejos en Ingeniería. Ciencia, Docencia y Tecnología, 30, (58). Disponible en: https://www.redalyc.org/articulo.oa?id=14560146012

Ochoviet, Cristina, \& Oktaç, Asuman (2011). Algunos aspectos del desarrollo del pensamiento algebraico: el concepto de raíz y de variable en ecuaciones polinómicas de segundo grado. Educación Matemática, 23(3),91-121. Disponible en: https://www.redalyc.org/articulo.oa?id=40521124005

Randolph, Valeria N., \& Parraguez, Marcela C. (2019). Comprensión del Sistema de los Números Complejos: Un Estudio de Caso a Nivel Escolar y Universitario. Formación universitaria, 12(6), 57-82. Disponible en https://dx.doi.org/10.4067/S0718-50062019000600057

Rodríguez, J. (2017). Métodos numéricos para la aproximación de raíces múltiples. Tesis Doctoral. Universidad de Salamanca, Disponible en. https://gredos.usal.es/bitstream/handle/10366/137629/TG_RORIGUEZ\%20LOP EZ\%2C\%20Juan\%20Manuel_Metodos\%20numericos.pdf?sequence=1\&isAllow ed $=y$ 


\section{PARA CITAR EL ARTÍCULO INDEXADO.}

Insuasti Castelo, R. M., \& Mendoza Castillo, J. R. (2021). Propuesta metodológica para la solución de ecuaciones polinómicas en el campo de los números complejos. ConcienciaDigital, $4(3.1)$ 291-300. https://doi.org/10.33262/concienciadigital.v4i3.1.1830

\section{Ciencia \\ LDigital}

El artículo que se publica es de exclusiva responsabilidad de los autores y no necesariamente reflejan el pensamiento de la Revista Conciencia Digital.

El artículo queda en propiedad de la revista y, por tanto, su publicación parcial y/o total en otro medio tiene que ser autorizado por el director de la Revista Conciencia Digital.

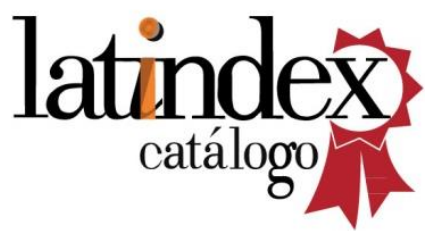

